\title{
Correlations between the SARS-COV-2 pandemic and increased aggressive behaviour to self and others
}

\author{
Laura Elena Bucur, Amira Chehab, Mirona Letiția Dobri, \\ Codrina Moraru, Petronela Nechita
}

\begin{abstract}
Laura Elena Bucur - Second Year Medical Resident in psychiatry, "Socola" Psychiatry Institute, Iași, Romania

Amira Chehab - Second Year Medical Resident in psychiatry, "Socola" Psychiatry Institute, Iași, Romania

Mirona Letiția Dobri - Fourth Year Medical Resident in psychiatry, "Socola” Psychiatry Institute, Iași, Romania

Codrina Moraru- Third Year Medical Resident in psychiatry, "Socola" Psychiatry Institute, Iași, Romania

Petronela Nechita - MD, PhD, Senior psychiatrist, "Socola” Psychiatry Institute, Iași, Romania
\end{abstract}

\begin{abstract}
The current times are facing an unprecedented phenomenon for our generations. The pandemic outbreak with SARS-CoV-2 rapidly and profoundly changed the aspect of the world we live in. The insecurity, the social isolation and its consequences, the fear of infection and the economic collapse are only a few of the elements that the current pandemic introduced in most lives. While the impact on physical health was immediately observed, the impact on mental health is gradually revealing itself and threatens to persist long-term. The socio-economic and psychological imbalances seem to leave a significant mark by triggering or enhancing mental disorders such as anxiety, depression, sleep disorders and posttraumatic stress disorder. All these are associated with an increased risk of aggressive behaviour to self and others, amplifying an already existing public health problem: suicide. Being defined as a self-harming behaviour based on the intention of ending one's life, suicide is responsible for approximately $\mathbf{8 0 0 0 0 0}$ deaths worldwide every year. Multiple social, economic and psychological factors are involved in this huge phenomenon and many of them are highly influenced by the current pandemic. Therefore raising awareness of the way pandemic-related changes might lead to suicide could be useful as a first step in elaborating prevention measures and avoiding tragedies.
\end{abstract}

KEYWORDS:

COVID-19, suicide, domestic violence, social isolation. 


\section{INTRODUCTION}

Aggressive behaviour is defined as a social conduct based on the intention of harming someone and it can be directed against others, being defined as hetero-aggressive behaviour, or against oneself, defined as auto-aggressive behaviour. The most severe form of self-aggression is suicide, constituting a current public health problem that affects all regions of the world, being a global phenomenon with a higher preponderance in the low- and middle-income countries. According to World Health Organization (WHO) in 2016 suicide was the second leading cause of death among 15-29 years old, with approximately 800000 cases worldwide every year. (1) Numerous risk factors are involved in the suicidal behaviour. While some of them, such as family history of suicide or preexisting psychiatric disorders cannot be modified, most risk factors can be highly influenced by external events, like the current SARS-CoV-2 pandemic, that started in December 2019 in Wuhan, China. Being one of the most challenging phenomena facing our world, the pandemic has led to several changes in multiple areas of our lives, and has had a high impact on socio-economical structure and both physical and mental health. Therefore it is very likely that the risk factors for suicide will be influenced by the current pandemic context and an impact over suicide rates and aggressive behaviour is expected. (2).

The aim of this paper is to raise awareness on how the current pandemic might intensify the risk factors for suicidal behaviour and domestic violence. Therefore we search to identify and describe the economic, social and psychological triggers of mental disorders that are associated with aggressive behaviour to self and others in the current pandemic context.

\section{THE PSYCHOLOGICAL IMPACT OF THE PANDEMIC ON MENTAL HEALTH AND THE ROLE OF MASS}

\section{MEDIA}

The widespread growth of the COVID-19 cases and the fact that the outbreak was rapidly declared a pandemic had a major psychological impact on the population: the fear of getting through the disease or of contracting and transmitting the virus, the social stigma that surrounded not only the infected but also the suspected ones, were often amplified by the media and social networks. The concern that grew along with the number of cases worldwide was overwhelming for some people, leading them to extreme gestures. A study conducted in China by Wang et al. at the beginning of the pandemic suggested that half of the participants rated their psychological impact as moderate to severe. $16.5 \%$ reported moderate to severe depressive symptoms, $28.8 \%$ reported moderate to severe anxiety symptoms, and $8.1 \%$ reported moderate to severe stress levels. $(3,4)$

Multiple cases of COVID-19 related suicides were reported starting from February 2020. The first case was reported in India, where a man who was returning to his village decided to hang himself in order to avoid spreading the virus throughout the village. Afterwards some other pandemic-related suicides were reported all over the globe. A 36-year old man in Bangladesh who presented fever killed himself while being prejudiced by his neighbors who were convinced that he had the virus. The tests performed postmortem revealed he was negative for SARS-CoV-2. (5) As a conclusion to these cases we can notice that the excessive fear of spreading the disease, sustained by the social stigma attached to those who might have COVID-19, can be a real and dangerous trigger for an altruistic suicide.

On the other hand, the overwhelming fear of the disease and its consequences might be a destabilizing element for some individuals. We support this statement with numerous examples of suicide cases that 
have happened all over the globe in the past few months. In England, a 19-year old girl took her life driven by unbearable concern regarding the impact that social isolation might have. In Saudi Arabia a Chinese student who was quarantined on suspicion of being infected with the coronavirus had committed suicide by jumping from the third floor of a hospital. (6) A 66-year old man with throat cancer hanged himself in a New York City hospital after testing positive for the novel coronavirus. An extreme example is the one of a man in Illinois who feared that his girlfriend contracted the virus and fatally shot her. Then he committed suicide. They both tested negative for COVID-19. (5) We highlight the fact that all these deaths were not caused by the viral infection itself, but directly by its psychological impact. The anxiety, the misinformation and the panic that grew along with the spread of the virus played a key role in the disproportional response of some individuals, who eventually found death more bearable than pandemic-related stress.

Both media and social networks generally play a key role in the public perception and they have a certain capacity of influencing people's behaviour. Despite being useful in informing the population about the current pandemic, media often proved itself imprudent. The exaggerated news reports and their continuous display often led to panic, distress and anxiety. Another study conducted in China by Dong and Zheng tried to evaluate the public perception over the COVID-19 news. It revealed that most people labeled the news as being exaggerated and overwhelming, suggesting that a better control over the circulating information is needed. (7) Moreover, the social networks were particularly involved in transmitting fake news, often leading to confusion and misinformation. The uncertainty, mistrust, the feeling of helplessness and indignation, together with a sense of losing control over their own lives were all intensified by contradictions and misleading information spread on several networks. All these brought their contribution to the imbalances everyone experiences nowadays, but they might have had a devastating impact on those with an already fragile emotional structure.(8) Therefore we cannot emphasize enough the importance of an ethical way of sharing pandemic-related news, considering that they might indirectly influence vulnerable categories, which are more prone to resort to extreme gestures such as suicide.

Another essential aspect that we should take into account when evaluating the impact of SARS-CoV-2 on suicide is that there is a high probability for survivors of severe forms of infection to be especially at risk of suicidal thoughts and behaviour. Because they experienced major stressors such as learning the diagnosis, the fear of transmitting the virus to their loved ones, the severe symptoms of infection, the hospital or even intensive care admission, these patients might be at high risk of developing depression and posttraumatic stress disorder. $(4,9)$ A study conducted in China by Bo et al. evaluated the mental state of patients infected with COVID-19 and it revealed that $96,2 \%$ of them had symptoms of posttraumatic stress disorder which are correlated with higher risk of suicidal thoughts and behaviour (10). Besides the psychological stress that infected patients are experiencing there is also a physio pathologic mechanism that is involved in the development of neuropsychiatric disorders. SARS-CoV-2 has tropism for central nervous system and the infection might lead to neurological disorders such as: ischemic stroke, seizures, encephalitis, dizziness, headache, ataxia and chronic pain. This is of high importance for our subject as these conditions are also correlated to a higher long-term suicide risk. (11) As a consequence, it is essential that we raise awareness over the importance of a proper support for these patients and of a higher 
vigilance on the possible display of psychiatric disorders associated with suicidal thoughts.

\section{THE SOCIAL ISOLATION AND ITS CONSEQUENCES}

Social distancing and isolation have become imperatives of our world since the SARS-CoV-2 outbreak. In order to limit the spread of the virus, the authorities worldwide recommended and even imposed a massive reduction of social contacts. Through direct measures such as the interdiction of gathering in large groups or even leaving the house during the lockdown, and indirect measures such as closing most of the public places where people had a chance to hang out, the authorities restricted more and more the social contact. This particular aspect is a double-edged sword. While from an epidemiological perspective these measures proved to be a highly effective method of limiting the spread of the virus, from a psychological point of view they might have a negative impact. In his iconic book, "Suicide", written in 1897, the sociologist Emile Durkheim emphases the major role of social factors, besides the psychological ones, in the mechanism of the suicidal act. He states that the absence of social integration of an individual is a key element in one's motivation of committing suicide.

A decrease in social cohesion and isolation will amplify the feeling of alienation and disconnect with subsequent depression, anxiety and eventually suicide (12). A study conducted by Stravynski et al., that followed the relation between loneliness and suicide attempts, proved there existed a strong connection between them. Defining an objective loneliness as for the ones who live alone, and a subjective loneliness as the feeling of alienation that one might feel, the study revealed that both of them are correlated with a higher prevalence of suicidal thoughts (13). From this perspective, we appreciate that the quarantine enforced by the current pandemic is associated with both types of loneliness: the objective one for those who live alone and also a subjective one through the affective echo that isolation from family and friends can have. Multiple studies have shown a higher prevalence of depression and anxiety among those who have experienced quarantine comparing to those who have not. As a parallel, during the SARS epidemic in Hong Kong in 2003, the social distancing played a role in the increasing suicide rates, for one third of the victims' experienced social confinement before taking their lives $(5,14)$. Although it is premature to evaluate the suicide prevalence related to the current pandemic, we think it might be useful to consider some aspects that might be correlated with a higher risk. Therefore Xiao et al. conducted a study including people who were under lockdown in the current pandemic context and it revealed that they had high levels of stress and anxiety and additional sleep disorders. We have previously mentioned the relationship between depression, anxiety and suicidal behaviour. In addition to that we highlight that insomnia and a poor quality of sleep are per se risk factors for suicidal thoughts. That is why an early and effective diagnosis and treatment of such disorders might save lives and a special attention should be given to them in the current context. (4, 15) Moreover staying in contact and maintaining the communication with family and friends through various means such as telephone might be an effective way of fighting loneliness and its terrible consequences as it might have a beneficial impact on those who are under lockdown.

\section{SELF-AGGRESSIVE BEHAVIOR IN RELATION TO HETERO- AGGRESSIVE BEHAVIOR IN THE CONTEXT OF SOCIAL ISOLATION}

A less discussed aspect of the isolation is that regarding an already vulnerable group: the victims of domestic violence. Domestic 
violence is defined as an abusive behaviour occurring in a family. According to the World Health Organization (WHO) it can take various forms, such as physical, psychological, economic or sexual violence. There is a multitude of behaviours that an aggressor can manifest and there is a "scale of violence", often beginning with intimidation, followed by isolation and devaluation of the victim and ending up in full-blown abuse. Therefore the isolation of the victim plays a key role in the display of domestic violence. Taking into account that the current pandemic context enforces social isolation in order to prevent the spread of SARS-CoV-2, an increase in the number of domestic violence cases is expected. (16) An explanation for this phenomenon also lies in the exacerbation of the risk factors and a decrease in the protective factors of domestic violence during lockdown. The forced and prolonged proximity between family members enhances the risk of domestic conflicts and an aggressive behaviour. Furthermore unemployment, financial difficulties and psychological distress due to pandemic may amplify the anger and hetero-aggressive impulses, exposing even more the victims. In the meantime a decrease of the protective factors is also a favorable element for manifestation of abuse. Isolation from family, friends and community will additionally increase the risk for victims while a more restricted access to the institutions that could support the abused ones will eventually condemn them. (17)

In France, the Minister of Interior reported a $30 \%$ raise of domestic violence during confinement since March 2020. This seems to be a global phenomenon as many countries reported a higher addressability to domestic violence hotlines since the pandemic outbreak: Spain reported a 20\%, England 25\%, Cyprus 30\% and Brazil up to $50 \%$ increase in the solicitations of hotlines. In addition, USA, Germany and Canada reported increasing cases of domestic violence. (18) A diametrically opposed phenomenon is witnessed in Italy, where a drop in the reported cases of domestic violence was registered during the lockdown. The decrease though does not reflect a real reduction in violent incidents, but a dangerous situation in which the abused ones encounter increased difficulties in asking for help from the community, while the aggressor gains more control over the victim. (16)

The impact that abuse can have over mental health is not at all neglectable. A study conducted by Brown and Seals, using data from the Kentucky Violent Death Reporting System from 2005-2015, reveals a strong correlation between domestic violence and suicide, reporting that $30 \%$ to $43 \%$ of suicides were committed after an abuse came from the partner (19). Also domestic violence is a trigger for some mental disorders such as mood disorders, anxiety, posttraumatic stress and addictions, all of them being associated with a higher suicidal risk. A highly affected group is composed of children, who are extremely susceptible to the tragic impact that abuse has on mental health. The trauma haunts them in their adult lives, predisposing them to several mental disorders, to social isolation, low self-esteem and suicidal behaviour (20). That is why facilitating access to institutions able to help victims during the lockdown should be a priority in order to avoid tragic consequences on short and long term.

\section{THE IMAPCT OF AN ECONOMIC CRISIS}

The restrictive measures enforced since the pandemic outbreak have had a major impact on the global economy. In order to limit the widespread of the virus numerous activities have been suspended. Auto isolation of both consumers and service providers, the closing of restaurants, limiting the artistic and cultural activities at the online environment are only few of 
the multiple reasons for which millions of people worldwide lost their jobs. Based on multiple observational studies the European Psychiatric Association (EPA) elaborated in 2016 a guidance paper which shows there is a strong connection between high unemployment rates and high suicide rates (21). The major financial crisis that the world has faced over time made the individuals liable to mental disorders such as depression, anxiety and suicide (5). For example during The Great Depression that USA faced during the '20s and ' 30 s, the massive economic collapse led to an increasing number in deaths by suicide (22). More recent data registered during the economic crisis between 2008 and 2010 sustain the connection between the two phenomena; studies showed that suicides committed out of financial reasons exceeded by 10000 the expected number for that period of time. (23)

Moreover a meta-analysis of prospective observational studies conducted by Kim and von dem Knesebeck, revealed that not only unemployment, but also the perceived job insecurity, the financial uncertainty associated with harsh times such as current pandemic may determine depression, anxiety, sleep disorders and alcohol abuse, eventually leading to suicide in many cases. (24) We cannot deny that nowadays uncertainty has become one of the most prevalent elements, and for many workers in various domains there persists a real threat of losing their jobs sooner or later. The distress generated by the constant and prolonged insecurity is not neglectable as it is a significant favorable factor for both auto-aggressive behavior and heteroaggressive behavior. In March 2020 The International Labor Organization estimated that a 5.3 to 24.7 million job loss increase is expected due to COVID-19. According to Kawohla and Nordta the unemployment rate is expected to register a raise of $4.936 \%$ to $5.644 \%$. These statistics can be translated by an increasing number of suicides of 2135 up to 9570 cases per year. $(6,25)$

These being said it is expected that the economic collapse that might come after the pandemic will have a major impact on the mental health causing depression, anxiety and eventually suicide in vulnerable individuals. Giving the fact that this is an adjustable and preventable factor we insist on the importance of elaborating efficient strategies that would facilitate the burden of poverty on the population and therefore lower the risk of deaths by suicide.

\section{CONCLUSIONS}

The SARS-CoV-2 pandemic is an unprecedented phenomenon for our generation and it brought rapid and profound changes in the socio-economic structure. For many people, it had a remarkable psychological impact, affecting not only the physical but also the mental health. The fear inspired by the infection with COVID-19, social distancing and isolation, unemployment and financial insecurity are some of the pandemic related changes that might precipitate or trigger mental disorders such as depression, anxiety and posttraumatic stress disorder. By leading to suicidal behaviour these conditions threaten to exacerbate this already existing health issue that affects hundreds of thousands of people worldwide. In addition, the social isolation, the high unemployment rates and the distress caused by the SARS-CoV-2 pandemic may increase the number of domestic violence cases by triggering aggressive behaviour within the family, and also by limiting the access to support for victims. Violence within family has long term consequences, predisposing the abused ones to self-aggressive behavior. Hence it is a priority to raise awareness on a large scale over the mechanisms and risk factors that can determine suicide and aggressive behaviour in the current pandemic context, in order to avoid further tragedies. 


\section{ACKNOWLEDGEMENTS AND DISCLOSURES}

The authors state that there are no declared conflicts of interest regarding this paper.

\section{REFERENCES}

1. https://www.who.int/news-room/fact-sheets/detail/suicide

2. Wasserman D, Iosue M, Wuestefeld A, Carli V. Adaptation of evidence-based suicide prevention strategies during and after the COVID-19 pandemic. World Psychiatry. 2020;19(3):294-306.

3. Wang C, Pan R, Wan X, et al. Immediate Psychological Responses and Associated Factors during the Initial Stage of the 2019 Coronavirus Disease (COVID-19) Epidemic among the General Population in China. Int J Environ Res Public Health. 2020;17(5):1729.

4. Sher L. COVID-19, anxiety, sleep disturbances and suicide. Sleep Med. 2020;70:124.

5. Sher L. The impact of the COVID-19 pandemic on suicide rates. QJM. 2020;113(10):707-712.

6. Kavukcu E, Akdeniz M. Tsunami after the novel coronavirus (COVID-19) pandemic: A global wave of suicide? International Journal of Social Psychiatry. July 2020.

7. Dong M, Zheng J. Letter to the editor: Headline stress disorder caused by Netnews during the outbreak of COVID-19. Health Expect. 2020;23(2):259-260.

8. Tagliabue F, Galassi L, Mariani P. The "Pandemic" of Disinformation in COVID-19 [published online ahead of print, 2020 Aug 1]. SN Compr Clin Med. 2020;1-3.

9. Sher L. Are COVID-19 survivors at increased risk for suicide? Acta Neuropsychiatr. 2020 Oct;32(5):270.

10. Bo HX, Li W, Yang Y, et al. Posttraumatic stress symptoms and attitude toward crisis mental health services among clinically stable patients with COVID-19 in China. Psychol Med. 2020;1-2.

11. Asadi-Pooya AA, Simani L. Central nervous system manifestations of COVID-19: A systematic review. J Neurol Sci. 2020;413:116832.

12. Durkheim E. Suicide. www.snowballpublishing.com 2013; 404.

13. Stravynski A, Boyer R. Loneliness in relation to suicide ideation and parasuicide: a population-wide study. Suicide Life Threat Behav. 2001;31(1):32-40.

14. Yip PS, Cheung YT, Chau PH, Law YW. The impact of epidemic outbreak: the case of severe acute respiratory syndrome (SARS) and suicide among older adults in Hong Kong. Crisis. 2010;31(2):86-92.

15. Xiao H, Zhang Y, Kong D, Li S, Yang N. Social Capital and Sleep Quality in Individuals Who Self-Isolated for 14 Days During the Coronavirus Disease 2019 (COVID-19) Outbreak in January 2020 in China. Med Sci Monit. 2020;26:e923921.

16. Sacco MA, Caputo F, Ricci P, et al. The impact of the Covid-19 pandemic on domestic violence: The dark side of home isolation during quarantine. Medico-Legal Journal. 2020;88(2):71-73.

17. Mazza M, Marano G, Lai C, Janiri L, Sani G. Danger in danger: Interpersonal violence during COVID-19 quarantine. Psychiatry Res. 2020;289:113046.

18. Ertan D, El-Hage W, Thierrée S, Javelot H, Hingray C. COVID-19: urgency for distancing from domestic violence. Eur J Psychotraumatol. 2020;11(1):1800245.

19. Brown S, Seals J. Intimate partner problems and suicide: are we missing the violence?. J Inj Violence Res. 2019;11(1):53-64.

20. Clarke A, Olive P, Akooji N, Whittaker K. Violence exposure and young people's vulnerability, mental and physical health. Int J Public Health. 2020;65(3):357-366.

21. Martin-Carrasco M, Evans-Lacko S, Dom G, et al. EPA guidance on mental health and economic crises in Europe. Eur Arch Psychiatry Clin Neurosci. 2016;266(2):89-124.

22. Tapia Granados JA, Diez Roux AV. Life and death during the Great Depression. Proc Natl Acad Sci U S A. 2009;106(41):17290-17295.

23. Reeves A, McKee M, Stuckler D. Economic suicides in the Great Recession in Europe and North America. Br J Psychiatry. 2014 Sep;205(3):246-7.

24. Kim TJ, von dem Knesebeck O. Perceived job insecurity, unemployment and depressive symptoms: a systematic review and meta-analysis of prospective observational studies. Int Arch Occup Environ Health. 2016;89(4):561-573.

25. Kawohl W, Nordt C. COVID-19, unemployment, and suicide. Lancet Psychiatry. 2020;7(5):389-390.

\section{Correspondence:}

Petronela Nechita,

$\mathrm{MD}, \mathrm{PhD}$, Senior psychiatrist, "Socola” Psychiatry Institute, Iași, Romania, craciunpetronela@yahoo.com 